\title{
Editorial: Immunogenic Cell Death in Cancer: From Benchside Research to Bedside Reality
}

\author{
Abhishek D. Garg* and Patrizia Agostinis* \\ Cell Death Research and Therapy (CDRT) Laboratory, Department of Cellular Molecular Medicine, KU Leuven University of \\ Leuven, Leuven, Belgium
}

Keywords: immunogenicity, cancer immunology, cancer immunotherapy, anticancer vaccines, damageassociated molecular patterns, danger signals, danger signalling, immunosurveillance

The Editorial on the Research Topic

Immunogenic Cell Death in Cancer: From Benchside Research to Bedside Reality

Immunogenic cell death (ICD) has emerged as a cornerstone of therapy-induced antitumor immunity (1-3). ICD is distinguished by spatiotemporally defined emission of danger signals or damageassociated molecular patterns (DAMPs) that elevate the immunogenic potential of dying cells [Garg et al.; (4)]. The important role played by DAMPs in immunity, tissue remodeling, and inflammation is discussed in details by Venereau et al. (Marco E. Bianchi lab).

Most potent ICD inducers, characterized so far, elicit danger signaling through oxidativeendoplasmic reticulum stress (5). Several ICD inducers have been characterized, e.g., some chemotherapies, some physicochemical therapies (e.g., radiotherapy or photodynamic therapy/PDT), and oncolytic viruses $(2,6)$. Here, radiotherapy is among the first recognized immunogenic therapies [on account of "abscopal-effect" (7)]. The immunogenic potential of radiotherapy and possibilities for its combination with immune checkpoint blockers is discussed by Derer et al. (Udo S. Gaipl lab). It is noteworthy that ICD can also be achieved by various "smart" combinatorial strategies - an important point for clinically applied non-ICD inducers, discussed in details by Bezu et al. (Guido Kroemer lab).

Several lines of experimental evidence have established the validity of ICD. However, the overreliance on usage of prophylactic vaccination in transplantable (heterotopic) tumor models has attracted some criticism (8). While these criticisms are valid, the field is already moving toward tumors produced orthotopically (curative/therapeutic) or in genetically engineered mouse models (GEMM) (at least for few ICD inducers, e.g., hypericin-PDT, Newcastle disease virotherapy and anthracyclines) (9-12). Moreover, the clinical existence of ICD has been proven through retrospective analysis involving cancer patient's survival/therapy-responsiveness data (13-17). These observations have encouraged the increased usage of ICD-associated DAMPs as predictive/prognostic biomarkers - a point discussed in detail by Fucikova et al. (Radek Spisek lab). The promising results generated by systemically administered ICD inducers have also paved way for application of ICD-based dendritic cell (DC) vaccines (12). This important development has been discussed from the preclinical/clinical vantage points of various solid tumors by Vandenberk et al. (Stefaan W. van Gool lab) and lymphoma by Zappasodi et al. (Massimo Di Nicola lab). In the latter case, it is clear that the field is moving toward chimeric antigen receptor (CAR)-T cell's application, and it will be interesting to see its combination with ICD in near future.

Nevertheless, the insurmountable complexity of cancer makes it inevitable that in certain contexts, ICD may fail. This failure may stem from various factors, e.g., tumor heterogeneity (8), MHC-level heterogeneity (12), pre-established niches enriched in immunosuppressive factors or immune-checkpoints (1), stem cell-based immune-evasion (12), low mutational load, inactivating 
mutations/polymorphisms in certain immune-receptors (1), general ablation of danger signaling (14), and other genetic or even epigenetic causes. Several of these pro-cancerous immuneevasive mechanisms and immunotherapeutic strategies required for overcoming them are discussed in detail by Kersten et al. (Karin E. de Visser lab). The strategies for targeting epigenetic processes to improve immunotherapy are further discussed by Wachowska et al. (Jakub Golab lab).

We believe that the valuable contributions of key researchers/ clinicians toward this research topic/special edition have largely fulfilled its primary aim, i.e., to foster a critical discussion on experimental and clinical relevance of ICD. In fact, to further summarize and organize the fields of ICD and DAMPs, we have produced a multi-author consensus paper within this research topic that attempts to classify DAMPs and ICD inducers with an eye on translational potential of ICD (Garg et al.). This classification paper brings together $>50$ authors from the fields of ICD and DAMPs, and tries to reach a comprehensive accord on various terminologies related to DAMPs/ICD, the historical background of these concepts, ICD classification system (Type I vs. Type II inducers), and the relevant preclinical/clinical criteria crucial for the field(s) (Garg et al.). We hope that this consensus paper will be a useful literature resource for various researchers/clinicians. These contributions, while summarizing the status quo, have also exposed a set of major questions and challenges that still need to be addressed.

\section{MAJOR QUESTIONS TO RESOLVE}

1. Which danger signaling module is most specific to ICD? Ecto-CRT seems to have remarkable exclusivity to ICD (10, 18-20) yet certain ICD inducers do not induce secreted-ATP (10), released-HMGB1 (19), or Type I IFN-responses (21). Alternatively, many non-ICD inducers induce secreted-ATP (22), released-HMGB1 (23), or Type I IFN-response (21). In fact, Type I IFN-responses can neutralize oncolytic viruses through antiviral signaling (24).

2. Are ICD-associated DAMPs interchangeable? Ecto-HSP90 was proposed to be interchangeable with ecto-CRT $(25,26)$, but this was recently invalidated in another set-up (21).

3. Could ICD-associated DAMPs act as bystanders in certain contexts? Induction of ICD-associated DAMPs may not always translate into a relevant functional outcome, e.g., Bleomycin induces all ICD-associated DAMPs yet elicits Tregs induction (27).

4. What is the full extent of "plasticity" of ICD-associated danger/ immunogenic signaling?

5. What is the exact role of cellular catabolic processes in regulating $I C D$ ? Current results are highly variable; while macroautophagy positively regulates secreted-ATP (28), yet it can also negatively regulate ecto-CRT (29-31). Also, the exact roles of chaperone-mediated autophagy/CMA [CMAessential gene Lamp2a regulates ecto-CRT (29)] or proteasome activity remains unresolved (Bortezomib induces ICD but not MG132, yet both inhibit the proteasome) (5).

6. What are the common molecular determinants of ICD across various cell death pathways? ICD-profile is largely associated with caspase-dependent apoptosis (18) but association with necroptosis is also emerging (10).

7. How does ICD counter-act the (innately) apoptosis-associated immunosuppressive processes?

8. Does the role of ROS in ICD extend beyond a proximal stressor? e.g., ROS-elicited oxidation-associated molecular patterns/OAMPs have been shown to mediate immunogenic potential (11).

9. Why ICD fails in certain (GEMM) cancer mice models (8) but works in others $(9,32)$ ?

10. Can epigenetic events [e.g., Long non-coding/micro-RNA (33)] regulate ICD and how?

\section{TRANSLATIONAL/CLINICAL CHALLENGES}

1. Can ICD's clinical translation withstand the "adverse effects" of mice-to-human immune differences?

2. Confirming ICD's existence in a prospective (high-powered/ supervised) clinical trial.

3. Can ICD withstand the (clinical-)operational/regulatory (GLP/ GMP/GCP) hurdles associated with anticancer vaccinesproduction? [indications for which are emerging (12)]

4. Characterizing ICD-resistance mechanisms in the clinic.

5. Characterizing reliable ICD-biomarker(s) detectable in patient tumor/sera-samples.

6. Investigating ICD as a source of robust prognostic/predictive/ mechanistic biomarkers [a point investigated recently in some studies $(13,34)]$.

We believe that the operational function of ICD (i.e., a dying cancer cell eliciting heightened immunogenicity-driven antitumor immunity) is incontrovertibly valid; but, owing to the incomprehensible complexity of cancer, the "specifics of ICD" (i.e., its molecular, signaling, and immunological determinants) will always remain open to amenability and variations. We envisage that overtime various "variants" of ICD may emerge that differ from each other in a manner dependent upon, the type of anticancer therapy, cancer cell death pathways, cancer-types, tumor antigen make-up, the in vivo/in situ location, and the location-dependent immune-contexture.

\section{AUTHOR CONTRIBUTIONS}

ADG wrote the manuscript. PA provided senior supervision and critically revised the manuscript.

\section{ACKNOWLEDGMENTS}

We would like to thank all the authors who contributed to this research topic as well as various reviewers/editors of the respective manuscripts, for their efforts, timely responses, and enthusiasm. We also thank the Frontiers Editorial Office for their assistance and support. This work was supported by $\mathrm{C} 1$ grant of the $\mathrm{KU}$ Leuven (C16/15/073), Federal Grant under the IAP7/32 of the Belgian Science Policy Office and FWO grant (G0584.12N) to PA. AG is supported by the FWO Postdoctoral Fellowship. 


\section{REFERENCES}

1. Kroemer G, Galluzzi L, Kepp O, Zitvogel L. Immunogenic cell death in cancer therapy. Annu Rev Immunol (2013) 31:51-72. doi:10.1146/ annurev-immunol-032712-100008

2. Kepp O, Senovilla L, Vitale I, Vacchelli E, Adjemian S, Agostinis P, et al. Consensus guidelines for the detection of immunogenic cell death. Oncoimmunology (2014) 3(9):e955691. doi:10.4161/21624011.2014.955691

3. Galluzzi L, Vacchelli E, Bravo-San Pedro JM, Buque A, Senovilla L, Baracco $\mathrm{EE}$, et al. Classification of current anticancer immunotherapies. Oncotarget (2014) 5(24):12472-508. doi:10.18632/oncotarget.2998

4. Kepp O, Galluzzi L, Martins I, Schlemmer F, Adjemian S, Michaud M, et al. Molecular determinants of immunogenic cell death elicited by anticancer chemotherapy. Cancer Metastasis Rev (2011) 30(1):61-9. doi:10.1007/s10555-011-9273-4

5. van Vliet AR, Martin S, Garg AD, Agostinis P. The PERKs of damageassociated molecular patterns mediating cancer immunogenicity: from sensor to the plasma membrane and beyond. Semin Cancer Biol (2015) 33:74-85. doi:10.1016/j.semcancer.2015.03.010

6. Adkins I, Fucikova J, Garg AD, Agostinis P, Spisek R. Physical modalities inducing immunogenic tumor cell death for cancer immunotherapy. Oncoimmunology (2014) 3:e968434. doi:10.4161/21624011.2014.968434

7. Kaminski JM, Shinohara E, Summers JB, Niermann KJ, Morimoto A, Brousal J. The controversial abscopal effect. Cancer Treat Rev (2005) 31(3):159-72. doi:10.1016/j.ctrv.2005.03.004

8. Ciampricotti M, Hau CS, Doornebal CW, Jonkers J, de Visser KE. Chemotherapy response of spontaneous mammary tumors is independent of the adaptive immune system. Nat Med (2012) 18(3):344-6. doi:10.1038/ nm. 2652

9. Michaud M, Xie X, Bravo-San Pedro JM, Zitvogel L, White E, Kroemer G. An autophagy-dependent anticancer immune response determines the efficacy of melanoma chemotherapy. Oncoimmunology (2014) 3(7):e944047. doi:10.416 $1 / 21624011.2014 .944047$

10. Koks CA, Garg AD, Ehrhardt M, Riva M, Vandenberk L, Boon L, et al. Newcastle disease virotherapy induces long-term survival and tumor-specific immune memory in orthotopic glioma through the induction of immunogenic cell death. Int J Cancer (2015) 136(5):E313-25. doi:10.1002/ijc.29202

11. Vandenberk L, Garg AD, Verschuere T, Koks C, Belmans J, Beullens M, et al. Irradiation of necrotic cancer cells employed for pulsing dendritic cells (DCs), potentiates DC vaccine-induced antitumor immunity against highgrade glioma. Oncoimmunology (2016) 5(2):e1083669. doi:10.1080/21624 02X.2015.1083669

12. Garg AD, Vandenberk L, Koks C, Verschuere T, Boon L, Van Gool S, et al. Dendritic cell vaccines based on immunogenic cell death elicit danger signals and T cell-driven rejection of high-grade glioma. Sci Transl Med (2016) 8(328):328ra27. doi:10.1126/scitranslmed.aae0105

13. Garg AD, De Ruysscher D, Agostinis P. Immunological metagene signatures derived from immunogenic cancer cell death associate with improved survival of patients with lung, breast or ovarian malignancies: a large-scale metaanalysis. Oncoimmunology (2016) 5(2):e1069938. doi:10.1080/2162402X. 2015.1069938

14. Garg AD, Elsen S, Krysko DV, Vandenabeele P, de Witte P, Agostinis P. Resistance to anticancer vaccination effect is controlled by a cancer cellautonomous phenotype that disrupts immunogenic phagocytic removal. Oncotarget (2015) 6(29):26841-60. doi:10.18632/oncotarget.4754

15. Apetoh L, Ghiringhelli F, Tesniere A, Obeid M, Ortiz C, Criollo A, et al. Toll-like receptor 4-dependent contribution of the immune system to anticancer chemotherapy and radiotherapy. Nat Med (2007) 13(9):1050-9. doi:10.1038/nm1622

16. Sistigu A, Yamazaki T, Vacchelli E, Chaba K, Enot DP, Adam J, et al. Cancer cell-autonomous contribution of type I interferon signaling to the efficacy of chemotherapy. Nat Med (2014) 20(11):1301-9. doi:10.1038/nm.3708

17. Vacchelli E, Ma Y, Baracco EE, Sistigu A, Enot DP, Pietrocola F, et al. Chemotherapy-induced antitumor immunity requires formyl peptide receptor 1. Science (2015) 350(6263):972-8. doi:10.1126/science.aad0779

18. Obeid M, Tesniere A, Ghiringhelli F, Fimia GM, Apetoh L, Perfettini JL, et al. Calreticulin exposure dictates the immunogenicity of cancer cell death. Nat Med (2007) 13(1):54-61. doi:10.1038/nm1523

19. Garg AD, Krysko DV, Verfaillie T, Kaczmarek A, Ferreira GB, Marysael T, et al. A novel pathway combining calreticulin exposure and ATP secretion in immunogenic cancer cell death. EMBO J (2012) 31(5):1062-79. doi:10.1038/ emboj.2011.497

20. Martins I, Kepp O, Schlemmer F, Adjemian S, Tailler M, Shen S, et al. Restoration of the immunogenicity of cisplatin-induced cancer cell death by endoplasmic reticulum stress. Oncogene (2011) 30(10):1147-58. doi:10.1038/ onc. 2010.500

21. Dudek-Peric AM, Ferreira GB, Muchowicz A, Wouters J, Prada N, Martin $\mathrm{S}$, et al. Antitumor immunity triggered by melphalan is potentiated by melanoma cell surface-associated calreticulin. Cancer Res (2015) 75(8):1603-14. doi:10.1158/0008-5472.CAN-14-2089

22. Martins I, Tesniere A, Kepp O, Michaud M, Schlemmer F, Senovilla L, et al. Chemotherapy induces ATP release from tumor cells. Cell Cycle (2009) 8(22):3723-8. doi:10.4161/cc.8.22.10026

23. Zhao T, Ren H, Jia L, Chen J, Xin W, Yan F, et al. Inhibition of HIF-1alpha by PX-478 enhances the anti-tumor effect of gemcitabine by inducing immunogenic cell death in pancreatic ductal adenocarcinoma. Oncotarget (2015) 6(4):2250-62. doi:10.18632/oncotarget.2948

24. Randall RE, Goodbourn S. Interferons and viruses: an interplay between induction, signalling, antiviral responses and virus countermeasures. JGen Virol (2008) 89(Pt 1):1-47. doi:10.1099/vir.0.83391-0

25. Zappasodi R, Pupa SM, Ghedini GC, Bongarzone I, Magni M, Cabras AD, et al. Improved clinical outcome in indolent B-cell lymphoma patients vaccinated with autologous tumor cells experiencing immunogenic death. Cancer Res (2010) 70(22):9062-72. doi:10.1158/0008-5472.CAN-10-1825

26. Fucikova J, Kralikova P, Fialova A, Brtnicky T, Rob L, Bartunkova J, et al. Human tumor cells killed by anthracyclines induce a tumor-specific immune response. Cancer Res (2011) 71(14):4821-33. doi:10.1158/0008-5472.CAN-11-0950

27. Bugaut H, Bruchard M, Berger H, Derangere V, Odoul L, Euvrard R, et al. Bleomycin exerts ambivalent antitumor immune effect by triggering both immunogenic cell death and proliferation of regulatory T cells. PLoS One (2013) 8(6):e65181. doi:10.1371/journal.pone.0065181

28. Michaud M, Martins I, Sukkurwala AQ, Adjemian S, Ma Y, Pellegatti P, et al. Autophagy-dependent anticancer immune responses induced by chemotherapeutic agents in mice. Science (2011) 334(6062):1573-7. doi:10.1126/ science. 1208347

29. Garg AD, Dudek AM, Agostinis P. Calreticulin surface exposure is abrogated in cells lacking, chaperone-mediated autophagy-essential gene, LAMP2A. Cell Death Dis (2013) 4:e826. doi:10.1038/cddis.2013.372

30. Martin S, Dudek-Peric AM, Maes H, Garg AD, Gabrysiak M, Demirsoy S, et al. Concurrent MEK and autophagy inhibition is required to restore cell death associated danger-signalling in Vemurafenib-resistant melanoma cells. Biochem Pharmacol (2015) 93(3):290-304. doi:10.1016/j.bcp.2014.12.003

31. Garg AD, Dudek AM, Ferreira GB, Verfaillie T, Vandenabeele P, Krysko $\mathrm{DV}$, et al. ROS-induced autophagy in cancer cells assists in evasion from determinants of immunogenic cell death. Autophagy (2013) 9(9):1292-307. doi:10.4161/auto.25399

32. Kroemer G, Galluzzi L, Zitvogel L. Immunological effects of chemotherapy in spontaneous breast cancers. Oncoimmunology (2013) 2(12):e27158. doi:10.4161/onci.27158

33. Musahl AS, Huang X, Rusakiewicz S, Ntini E, Marsico A, Kroemer G, et al. A long non-coding RNA links calreticulin-mediated immunogenic cell removal to RB1 transcription. Oncogene (2015) 34(39):5046-54. doi:10.1038/ onc. 2014.424

34. Stoll G, Bindea G, Mlecnik B, Galon J, Zitvogel L, Kroemer G. Meta-analysis of organ-specific differences in the structure of the immune infiltrate in major malignancies. Oncotarget (2015) 6(14):11894-909. doi:10.18632/ oncotarget. 4180

Conflict of Interest Statement: The authors declare that the research was conducted in the absence of any commercial or financial relationships that could be construed as a potential conflict of interest.

Copyright (C) 2016 Garg and Agostinis. This is an open-access article distributed under the terms of the Creative Commons Attribution License (CC BY). The use, distribution or reproduction in other forums is permitted, provided the original author(s) or licensor are credited and that the original publication in this journal is cited, in accordance with accepted academic practice. No use, distribution or reproduction is permitted which does not comply with these terms. 